\title{
80.
}

\section{SUR QUELQUES TRANSMUTATIONS DES LIGNES COURBES.}

[From the Journal de Mathématiques Pures et Appliquées (Liouville), tom. xIv. (1849) pp. $40-46$.

JE suppose qu'une courbe quelconque soit représentée par une équation homogène entre trois variables $x, y, z$, et je me propose d'examiner (en ne faisant attention qu'aux droites et aux coniques) ce que signifient les transmutations
I.$$
\xi=\sqrt{x}, \quad \eta=\sqrt{y}, \quad \zeta=\sqrt{z},
$$
II.

$$
\xi=x^{2}, \quad \eta=y^{2}, \quad \zeta=z^{2},
$$
III.

$$
\xi=\frac{1}{x}, \quad \eta=\frac{1}{y}, \quad \xi=\frac{1}{z},
$$

ces quantités étant prises dans chaque cas pour de nouvelles coordonnées. Les théorèmes auxquels on se trouve conduit comprennent les théorèmes qu'obtient M. William Roberts par sa méthode générale de transmutation en écrivant $n=\frac{1}{2}, n=2$. (Voir sa Note sur ce sujet, t. xIII. [1848] de ce Journal, page 209). ${ }^{1}$

\section{Soit}

$$
\xi=\sqrt{x}, \quad \eta=\sqrt{y}, \quad \xi=\sqrt{z} .
$$

Je supposerai ici et partout dans ce mémoire que $P Q R$ soit le triangle formé par les droites

$$
x=0, \quad y=0, \quad z=0 .
$$

1 M. William Roberts a bien voulu me parler l'été passé à Dublin, avant que cette Note eût paru, des théorèmes qui devaient s'y trouver: cela me suggéra, et je lui communiquai, peu de jours après, la deuxième et la troisième de mes méthodes de transmutation. On verra dans la suite que ce sont la première et la deuxième de mes méthodes qui correspondent aux cas $n=\frac{1}{2}, n=2$, respectivement, mais que la troisième est aussi très étroitement liée avec le cas $n=\frac{1}{2}$. 
Cela posé, en considérant les nouvelles coordonnées $\xi, \eta, \zeta$, on voit tout de suite qu'un système d'équations telles que

$$
\xi: \eta: \zeta=\alpha: \beta: \gamma
$$

correspond à un point; qu'une équation linéaire quelconque

$$
A \xi+B \eta+C \zeta=0
$$

correspond à une conique qui touche les trois côtés du triangle $P Q R$ (ou, si l'on veut, inscrite à ce triangle); et qu'une équation quelconque du second ordre

$$
a \xi^{2}+b \eta^{2}+c \xi^{2}+2 f \zeta \eta+2 g \xi \zeta+2 h \eta \xi=0
$$

correspond à une courbe du quatrième ordre qui touche les trois côtés du triangle $P Q R$, chaque côté deux fois. Et réciproquement, de telles coniques et de telles courbes du quatrième ordre peuvent toujours se représenter par une équation linéaire ou par une équation du second ordre entre les coordonnées $\xi, \eta$, $\zeta$.

On déduit de là cette propriété générale:

"Tout théorème descriptif qui se rapporte à des points, à des droites, et à des coniques, conduit à un théorème qui se rapporte d'une manière analogue à des points, à des coniques qui touchent chacune trois droites fixes, et à des courbes du quatrième ordre qui touchent chacune ces trois droites fixes, deux fois chaque droite."

En supposant que les coefficients $g, h$ se réduisent à zéro, l'équation

$$
a \xi^{2}+b \eta^{2}+c \zeta^{2}+2 f \eta \zeta=0
$$

est celle d'une conique tangente aux deux droites $P Q, P R$, et la courbe du quatrième ordre se réduit à deux coniques égales et superposées l'une à l'autre. De là:

"Tout théorème descriptif qui se rapporte à des points, à des droites, et à une conique, conduit à un théorème qui se rapporte d'une manière analogue à des points, à des coniques qui touchent chacune trois droites fixes, et à une conique qui touche deux de ces droites fixes."

En particulier, en supposant que les deux droites fixes que la conique touche soient les deux droites tangentes à cette conique qui passent par un des foyers, et que la troisième droite fixe soit à l'infini:

"Tout théorème descriptif qui se rapporte à des points, à des droites, et à une conique, conduit à un théorème qui se rapporte d'une manière analogue à des points, à des paraboles qui ont pour foyer commun un point fixe, et à une conique qui a aussi ce point fixe pour un de ses foyers."

II. Soit à présent

$$
\xi=x^{2}, \quad \eta=y^{2}, \quad \zeta=z^{2} .
$$

Un système d'équations telles que

$$
\xi: \eta: \zeta=\alpha: \beta: \gamma
$$


correspond à quatre points qui formeront ce que l'on peut nommer un système symétrique par rapport aux points $P, Q, R$, ou tout simplement un système symétrique. On voit sans peine que les quatre points d'un même système symétrique sont tels, que le quadrilatère formé par ces quatre points a pour points de concours des diagonales et des côtés opposés les points $P, Q, R$. Pour ne pas interrompre la suite'des raisonnements, il convient d'entrer dans quelques détails relatifs à ce sujet. Nous nommerons courbe symétrique par rapport aux points $P, Q, R$, ou simplement courbe symétrique, toute courbe lieu d'un système symétrique. Cela posé, il y a une infinité de coniques symétriques; savoir, toute conique par rapport à laquelle les points $P, Q, R$ sont des points conjugués (cela veut dire par rapport à laquelle l'un quelconque de ces points a pour polaire la droite menée par les deux autres) est une conique symétrique. Remarquons qu'une courbe symétrique du quatrième ordre peut se réduire à un système de deux coniques telles, que les points de chaque système symétrique de la courbe soient partagés deux à deux sur les deux coniques. En effet, considérons une conique telle, que, par rapport à cette conique, le point $P$ ait pour polaire la droite $Q R$. Il est facile de construire une autre conique telle, que l'ensemble des deux coniques soit une courbe symétrique. Pour cela, menons une transversale quelconque $P M N$ qui rencontre la conique donnée aux points $M, N$ : soient $M^{\prime}, N^{\prime}$ les points de rencontre des droites $Q M, N R$ et des droites $Q N, M R$; le lieu des deux points $M^{\prime}, N^{\prime}$ (lesquels seront en ligne droite avec le point $P$ ) sera la conique dont il s'agit. Nous dirons que les deux coniques sont des coniques supplémentaires.

En revenant à notre but actuel, une équation linéaire quelconque

$$
A \xi+B \eta+C \zeta=0
$$

correspond à une conique symétrique. Une équation du second ordre quelconque

$$
a \xi^{2}+b \eta^{2}+c \zeta^{2}+2 f \eta \zeta+2 g \zeta \xi+2 h \xi \eta=0
$$

correspond à une courbe symétrique du quatrième ordre. Et réciproquement, toute conique symétrique, ou toute courbe symétrique du quatrième ordre, peut se représenter par une équation linéaire, ou du second ordre, avec les coordonnées $\xi, \eta$, $\zeta$. De là cette propriété générale:

"Tout théorème descriptif qui se rapporte à des points, à des droites, et à des coniques, conduit à un théorème qui se rapporte d'une manière analogue à des systèmes symétriques (par rapport à trois points fixes), à des coniques symétriques, et à des courbes symétriques du quatrième ordre."

Dans ce théorème on peut, si l'on veut, considérer l'un des points d'un système symétrique comme représentant le système, et substituer aux mots systèmes symétriques (par rapport à trois points fixes), le mot points.

En supposant que dans la courbe du quatrième ordre on ait à la fois

$$
a c-g^{2}=0, \quad a b-h^{2}=0,
$$

il est facile de voir que la courbe du quatrième ordre se réduit à deux coniques supplémentaires.

C. 
En effet, ces conditions étant remplies, en rétablissant les valeurs de $\xi, \eta$, $\zeta$, on obtient une équation qui se divise en deux équations, telles que

$$
\alpha x^{2}+\beta y^{2}+\gamma z^{2}-\delta y z=0, \quad \alpha x^{2}+\beta y^{2}+\gamma z^{2}+\delta y z=0,
$$

qui appartiennent, comme on le voit sans peine, à une paire de coniques supplémentaires.

De là, en remarquant qu'il est permis de faire abstraction de l'une de ces coniques:

"Tout théorème qui se rapporte à des points, à des droites, et à une conique, conduit à un théorème qui se rapporte d'une manière analogue à des points, à des coniques symétriques (par rapport à trois points fixes), et à une conique telle, que, par rapport à cette conique, l'un des trois points fixes a pour polaire la droite menée par les deux autres points fixes."

Supposons en particulier que les deux points fixes dont nous venons de parler soient les points où la droite à l'infini est rencontrée par un cercle qui a pour centre le troisième point fixe; ce troisième point fixe sera le centre tant des coniques symétriques par rapport à ces trois points fixes, que de la conique par rapport à laquelle le troisième point fixe a pour polaire la droite menée par les deux autres points fixes. De plus, les asymptotes de l'une quelconque des coniques symétriques formeront avec les droites imaginaires asymptotes du cercle un faisceau harmonique, et de là les asymptotes de la conique dont il s'agit seront à angle droit, ou, autrement dit, cette conique sera une hyperbole équilatère. De là enfin:

"Tout théorème descriptif qui se rapporte à des points, •à des droites, et à une conique, conduit à un théorème qui se rapporte d'une manière analogue à des points, à des hyperboles équilatères et concentriques, et à une conique concentrique avec les hyperboles."

III. Soit enfin

$$
\xi=\frac{1}{x}, \quad \eta=\frac{1}{y}, \quad \xi=\frac{1}{z} .
$$

Cette supposition conduit à l'une des méthodes de transmutation données par M. Steiner parmi les observations générales qui forment la conclusion de son ouvrage intitulé: Systematische Entwickelung \&c., [Berlin, 1832], méthode qu'obtient M. Steiner au moyen de la théorie de l'hyperboloïde gauche. Je la reproduis ici tant pour en faire voir la théorie analytique qu'à cause de son analogie avec les méthodes I. et II.

Il est évident d'abord qu'un système d'équations telles que

$$
\xi: \eta: \zeta=\alpha: \beta: \gamma
$$

correspond à un point; de même, une équation linéaire quelconque, telle que

$$
A \xi+B \eta+C \zeta=0 \text {, }
$$

correspond à une conique qui passe par les trois points $P, Q, R$, et une équation quelconque du second ordre, telle que

$$
a \xi^{2}+b \eta^{2}+c \zeta^{2}+2 f \eta \zeta+2 g \zeta \xi+2 h \xi \eta=0
$$


correspond à une courbe du quatrième ordre qui a les trois points $P, Q, R$ pour points doubles. Réciproquement, de telles coniques et de telles courbes du quatrième ordre peuvent toujours se représenter par des équations linéaires et par des équations du second ordre. De là :

"Tout théorème descriptif qui se rapporte à des points, à des droites, et à des coniques, conduit à un théorème qui se rapporte d'une manière analogue à des points, à des coniques qui passent par trois points fixes, et à des courbes du quatrième ordre qui ont ces trois points fixes pour points doubles."

En supposant que l'on ait à la fois $a=0, b=0$, la courbe du quatrième ordre se réduit à une conique qui passe par les deux points $Q, R$ (ou, si l'on veut, 'au système formé de cette conique et des droites $P Q, P R)$. De là :

"Tout théorème descriptif qui se rapporte à des points, à des droites, et à une conique, conduit à un théorème qui se rapporte d'une manière analogue à des points, à des coniques qui passent par trois points fixes, et à une conique qui passe par deux de ces points fixes."

On ne peut pas particulariser ce théorème de manière à obtenir des théorèmes intéressants. Mais, en prenant les polaires réciproques des deux théorèmes qui viennent d'être obtenus, on a ces propriétés nouvelles:

"Tout théorème descriptif qui se rapporte à des droites, à des points, et à des coniques, conduit à un théorème qui se rapporte d'une manière analogue à des droites, à des coniques qui touchent chacune trois droites fixes, et à des courbes de la quatrième classe qui touchent chacune ces mêmes droites fixes, deux fois chaque droite;"

et:

"Tout théorème qui se rapporte à des droites, à des points, et à une conique, conduit à un théorème qui se rapporte d'une manière analogue à des droites, à des coniques qui touchent chacune trois droites fixes, et à une conique qui touche deux de ces droites fixes."

De là, comme dans la méthode I.:

"Touit théorème qui se rapporte à des droites, à des points, et à une conique, conduit à un théorème qui se rapporte d'une manière analogue à des droites, à des paraboles qui ont pour foyer commun un point fixe, et à une conique qui a ce même point fixe pour un de ses foyers;"

théorème que l'on doit comparer avec le troisième théorème que donne la méthode I.

Pour compléter cette théorie, nous aurions dû ajouter les deux théorèmes polaires réciproques du premier et du deuxième théorème que donne la méthode I.; mais cela se fait sans la moindre peine. Quant au premier et au deuxième théorème que donne la méthode II., les polaires réciproques de ces deux théorèmes ne conduisent à rien de nouveau. 